\title{
Intelectin-1 and Endocrinological Parameters in Women with Polycystic Ovary Syndrome: Effect of Insulin Resistance
}

\author{
Adnan Jassim Mohammed Al-Fartosy, Nadhum Abdulnabi Awad, Amel Hussein Mohammed \\ Department of Chemistry, College of Science, University of Basra, Basra, Iraq
}

Objectives: We aimed to evaluate the effect of insulin resistance (IR) on serum Intelectin-1 and endocrinological hormones levels in obese and non-obese women with and without polycystic ovary syndrome (PCOS) in Basrah, Iraq.

Methods: From 124 women volunteers, 60 patients with primary and 64 patients with secondary, while 56 normal ovulatory women were taken as controls. Their fasting insulin hormone, intelectin-1, anti-Mullerian hormone, luteinizing hormone (LH), follicle stimulating hormone (FSH), prolactin (PRL), estradiol (E2) and testosterones (T) were determined by ELISA methods. BMI, glucose and quantitative insulin sensitivity check index as well as IR was determined by the homeostasis model assessment.

Results: A significant changes $(\mathrm{P}<0.05)$ were seen in the level of homeostasis model assessment-IR, E2 and T. Levels of anti-Mullerian hormone, LH, LH/FSH ratio and prolactin were significantly $(\mathrm{P}<0.01)$ increased and level of intelectin- 1 and $\mathrm{E} 2 / \mathrm{T}$ ratio were significantly $(\mathrm{P}<0.01)$ decreased, while quantitative insulin sensitivity check index level was not significantly different $(\mathrm{P}>0.05)$ between the patients $\left(1^{\circ} \mathrm{PCOS}\right.$ and $\left.2^{\circ} \mathrm{PCOS}\right)$ and control groups. On the other hand, our data reported that FSH level was significantly $(\mathrm{P}<0.05)$ lower in obese and higher in non-obese patients with PCOS as compared to control group.

Conclusion: Levels of intelectin-1 and endocrinological hormones have significantly associated with body mass index, IR and physical activity in patients and normal groups and the strategies that can modulate levels of these parameters would improve metabolic disarrangements in women with PCOS. (Ewha Med J 2020;43(1):1-11)

\author{
Received September 23, 2019 \\ Revised November 13, 2019 \\ Accepted November 18, 2019 \\ Corresponding author \\ Adnan Jassim Mohammed Al-Fartosy \\ Department of Chemistry, College of Science, \\ University of Basra, Qarmet Ali, Basra 61001, \\ Iraq \\ Tel: 964-780-2869-507, Fax: 964-771-0809- \\ 414 \\ E-mail: adnan.Jassim@uobasrah.edu.iq
}

Key Words

Intelectin-1; Body mass index; Insulin resistance; Gonadal steroid hormones; Polycystic ovary syndrome

\section{Introduction}

The adipose tissue body mass may play a critical and essential role in normal development of women reproductive functions. Also, the obesity has been shown to produce derangement of women reproductive functions and infertility. It is one of the most striking findings in polycystic ovary syndrome (PCOS) related to insulin resistance (IR) which is characterized as visceral and abdominal subcutaneous adipose tissue accumulation and known that $30 \%$ to $60 \%$ of all PCOS patients are overweight or obese [1]. PCOS is the most common endocrinopathy amongst women of reproductive age, with prevalence up to $15 \%$. It is a heterogeneous hormonal and metabolic disorder with only partially explained pathogenesis. On the other hand, it has been shown that IR, hyperinsulinemia and hyperandrogenism are improved in PCOS patients who lose 5\% to 7\% of their basal weight, which seem to be the important links of PCOS pathogenesis in at least some causes.

This is an Open Access article distributed under the terms of the Creative Commons Attribution Non-Commercial License (http://creativecommons.org/licenses/by-nc/4.0) which permits unrestricted non-commercial use, distribution, and reproduction in any medium, provided the original work is properly cited. 
The PCOS pathogenesis remains largely unknown but it mainly has been attributed to the disorder of folliculogenesis which causing oligo-ovulatory cycles or impaired in folliculogenesis with increased preantral and small antral follicle counts, which lead to the increase in levels of anti-Mullerian hormone (AMH) [2]. In PCOS the IR may be due in part to the high prevalence of obesity in those with the disorder. Therefore, controversy still exists regarding whether it results from the concurrent obesity or from PCOS. It is possible that adipose tissue dysfunction may play a main role in the observed IR and, consequently, to the metabolic and cardiovascular consequences of the disorder.

Adipose tissue is an endocrine organ that has widespread effects on carbohydrate and lipid metabolism. Furthermore, evidence exists for altered adipocyte function in PCOS, with studies reporting altered secretion of adipokines [3]. Some adipokines are produced mainly in adipose tissue, but not exclusively, by adipocytes, while others are partially produced by infiltrating macrophages and stromal cells. The imbalance of production of these adipokines may cause the development of obesity related metabolic and vascular disorders.

Human intelectin-1 ("intestinal lectin”, also named as omentin-1, endothelial lectin $\mathrm{HL}^{-1}$ and intestinal lactoferrin receptor) is a novel fat depot-specific $34-\mathrm{kDa}$ secretory protein that was first identified by its ability to bind galactofuranose units in the carbohydrate chains of bacterial cell walls [4]. It is a new type of $\mathrm{Ca}^{2+}$-dependent lectin with affinity for galacto furanosyl residues (constituents of pathogens and dominant inmunogens). Intelectin-1 was identified from a cDNA library of visceral omental adipose tissue by Tang et al. [5] and is preferentially produced by visceral adipose tissue with compared to subcutaneous adipose tissue. It was also identified in human epicardial fat. Subsequently, intelectin-1 was reported to be highly expressed in the visceral adipose tissue, especially in stromal vascular cells and to a lesser extent in adipocytes. In vitro experiments have revealed that treatment with recombinant intelectin-1 enhanced insulin-stimulated glucose uptake in human subcutaneous and omental adipocytes, triggering activating protein kinase B (also known as Akt) signaling in absence and the presence of insulin [2].

Even though the scientific community has started unraveling the mysteries of the close linkage between obesity, IR, hormones and their physiological effects, a lot still remains to be discovered. In the province of Basrah (southern of Iraq), to date, no study has investigated on obesity, IR and its effects on PCOS women patients. Present study is, therefore, centered on the objective of evaluating the effect of IR on serum intelectin-1 and endocrinological hormones levels in obese and non-obese women with and without PCOS in Basrah province (southern of Iraq).

\section{Methods}

\section{Subjects}

The present study is an interventional prospective randomized controlled clinical trial. Samples were collected from infertility center at Basra Hospital for Obstetrics and Children in Basrah province-Iraq during the period from December 2016 till end of January 2019. In addition to patient samples obtained from a private clinics run by consultant professor Dr. Abdulkareem Hussain Sabar and assistant professor Dr. Nateek Dicran Caspar in Obstetrics and Infertility Medicine. In this study, 284 women volunteers were participated in the study.

One hundred and twenty-four of women patients with PCOS, 60 patients with primary (couple who had no previous pregnancies for at least one year after marriage, 28 obese and 32 non-obese) and 64 patients with secondary (couple who have conceived previously, although the pregnancy may not have been successful for example, miscarriage, and ectopic pregnancy, 31 obese and 33 non-obese), and 56 normal ovulatory women (fertile women attending the hospital with a genital prolapsed and history of at least one childbirth were taken as controls, 27 obese and 29 non-obese) were followed up for 14 months, till end the study. While, 104 of women volunteers (70 patients and 34 healthy controls) were excluded from the study due to enable to follow up study. The patients are already diagnosed as PCOS women according to the basis of American College of Obstetricians and Gynecologists [6].

All the participants were of Basra governorate (Southern of Iraq). The sample populations are married women who live together with her husband and have not used any contraceptive method for a period of one year, but they are still childless. The controls were volunteers who freely agreed to participate in the study. The participants were in the age group of $18-45$ years for patients and of 20-45 years for healthy control.

Exclusion factors were diseases affecting the metabolic state 
or not suitable to participate in this study. Women presenting endometriosis, uterine fibroid, breast cancer, epilepsy or migraine and those with hormone-dependent cancer were excluded. Furthermore, hyper- and hypo-thyroidism, diabetes, mental disease, serious disease with dysfunction of heart, liver, kidney, were excluded as well as that using estrogen replacement therapy. The control group had regular menstrual cycles (26-30 day) and not used oral contraceptives for at least the preceding 3 months and had no clinical signs of hyperandrogenemia or any sign of PCOS symptoms.

The study received an ethical approval from Basrah University (7/54/4926), and an informed consent was obtained from each participant after explanation of the procedures in full detail. The informed consent and ethical guidelines were followed, based on the Deceleration of Helsinki for year 2000.

\section{Samples}

All blood samples were obtained in the morning between 09:00 and 09:30 hours after a 12-hour fast and a 30-minute of rest in the supine position. Blood was collected at 2 or 3 days of menstrual cycle, disposable plastic syringes and needles were used for blood collection.

Venous fasting blood samples $(10 \mathrm{~mL})$ were collected from patients and healthy volunteers by vein puncture, then transferred to plain tube (without anticoagulant) which allowed clotting in a clean plain tube for 30 minutes at room temperature. After the blood had clotted it was placed in a centrifuge and spun at $402 \times \mathrm{g}$ for 10 minutes to obtain the serum.

The obtained serum immediately use in detection of variables in this study, and others were stored in deep freezing at $-20^{\circ} \mathrm{C}$ until using.

\section{Methods of biochemical estimation}

The control and PCOS patient's blood samples were analyzed for biochemical parameters by standard procedures as follows: body mass index (BMI) was calculated as the following formula (BMI $\left[\mathrm{kg} / \mathrm{m}^{2}\right]=$ weight $[\mathrm{kg}] /$ height $\left.\left[\mathrm{m}^{2}\right]\right)$ [7], serum glucose was estimated by kit (KA0831, Abnova, Taipei, Taiwan), serum intelectin-1 level was determined using the (E0155Hu, Bioassay Technology Laboratory, Shanghai, China) kit which was a solid phase ELISA based on the sandwich principle. AMH was estimated by kit (E-EL-H0317, Elabscience, Houston, TX, USA), insulin was estimated by kit
(KA0921, Abnova), follicle stimulating hormone (FSH) was estimated by kit (KA0213, Abnova), luteinizing hormone (LH) was estimated by kit (KA0214, Abnova), prolactin (PRL) was estimated by kit (KA0217, Abnova), testosterone (T) was estimated by kit (KA0236, Abnova), and estradiol (E2) was estimated by kit (KA0234, Abnova).

IR was determined by the homeostasis model assessment [HOMA-IR=insulin $(\mu \mathrm{IU} / \mathrm{mL}) \times$ glucose $(\mathrm{mg} / \mathrm{dL}) / 405]$ [7], quantitative insulin sensitivity check index [QUICKI $=1-\{(\log$ insulin, $\mu \mathrm{IU} / \mathrm{mL})+(\log$ glucose, $\mathrm{mg} / \mathrm{dL})\}][7]$.

\section{Statistical analysis}

We used Student t-test and Pearson's correlation coefficient to find the statistical significance. A P-value $\langle 0.05$ was to be considered statistically significant and $\mathrm{P}<0.01$ for highly significant.

\section{Results}

The general characteristics of all subjects participated in the present study were presented in in Table 1. Compared with normal controls, the results indicated that patients women with $1^{\circ} \mathrm{PCOS}$ and $2^{\circ} \mathrm{PCOS}$ (both group of obese and non-obese) had increased levels of HOMA-IR ( $\mathrm{P}<0.05), \mathrm{AMH}(\mathrm{P}<0.01)$, $\mathrm{LH}(\mathrm{P}<0.01), \mathrm{LH} / \mathrm{FSH}$ ratio $(\mathrm{P}<0.01)$, E2 (P<0.05), T $(\mathrm{P}<0.05)$ and PRL $(\mathrm{P}<0.01)$, as shown in Table 2.

Furthermore, same Table 2 reflect that patients with PCOS had lower intelectin-1 $(\mathrm{P}<0.01)$ and $\mathrm{E} 2 / \mathrm{T}$ ratio $(\mathrm{P}<0.01)$, while QUICKI level was not significantly different $(\mathrm{P}\rangle 0.05)$ between the patients $\left(1^{\circ} \mathrm{PCOS}\right.$ and $\left.2^{\circ} \mathrm{PCOS}\right)$ and control groups. On the other hand, our data reported that FSH level was significantly $(\mathrm{P}<0.05)$ lower in obese and higher in non-obese patients with PCOS as compared to control group.

\section{Discussion}

To the best of our knowledge, this is the first study embarked on the objective of evaluating the effect of IR on serum intelectin-1 and endocrinological hormones levels in obese and nonobese women with and without PCOS in Basrah province (southern of Iraq).

In the present study, data showed that more than half of the PCOS patients were nonsmokers. Also, most of the volunteers 
Table 1. The demographic characteristics of the present study

\begin{tabular}{|c|c|c|c|}
\hline \multirow[b]{2}{*}{ Characteristics } & \multirow[b]{2}{*}{ Control } & \multicolumn{2}{|c|}{ Women patients with PCOS } \\
\hline & & $\begin{array}{c}\text { Primary } \\
\left(1^{\circ} \mathrm{PCOS}\right)\end{array}$ & $\begin{array}{c}\text { Secondary } \\
\left(2^{\circ} \mathrm{PCOS}\right)\end{array}$ \\
\hline \multirow[t]{2}{*}{ Total } & 56 & \multicolumn{2}{|c|}{124} \\
\hline & & 60 & 64 \\
\hline Age (yr) & $30.44 \pm 4.84$ & $28.88 \pm 6.64$ & $29.72 \pm 4.62$ \\
\hline BMI $\left(\mathrm{kg} / \mathrm{m}^{2}\right)$ & $26.73 \pm 2.54$ & $27.85 \pm 1.98$ & $27.685 \pm 2.06$ \\
\hline \multicolumn{4}{|l|}{ Smoking habit } \\
\hline Negative & 49 & 52 & 58 \\
\hline Positive & 7 & 8 & 6 \\
\hline \multicolumn{4}{|l|}{ Demographic area } \\
\hline Urban & 50 & 56 & 57 \\
\hline Rural & 6 & 4 & 6 \\
\hline \multicolumn{4}{|l|}{ Education } \\
\hline Learned & 45 & 51 & 49 \\
\hline Illiterate & 11 & 9 & 15 \\
\hline \multicolumn{4}{|l|}{ Employment } \\
\hline Employed & 41 & 43 & 44 \\
\hline Not employed & 15 & 17 & 20 \\
\hline
\end{tabular}

Values are presented as number or mean \pm SD.

PCOS, polycystic ovary syndrome; BMI, body mass index.

from both patients' women with PCOS and healthy control were from urban, all of them received a well-learned and also they had a good employed place, as shown in Table 1. The major differences between urban and rural areas are the differences in pollution, environments, social, psychological, genetic, food factors and others, which are increasing dramatically in urban areas [8]. On the other hand, the psychological of women may be affected by work pressures and its requirements and household factors such as child's-rearing and marital relationships that are often tense and not good which are increased the imbalance of oxidant/antioxidant [9].

Intelectin-1 is a novel fat depot-specific secretory protein and this adipokine has an ability to improve and increases the insulin sensitivity through improved insulin-stimulated glucose transport and Akt phosphorylation and is expressed in visceral adipose tissue that was identified from a cDNA library of visceral omental adipose tissue by Yang et al. in the year of 2006 [10]. In the present study, significantly decreased level of intelectin-1 in PCOS women could be attributed to the higher levels of serum glucose, insulin hormone, IR and androgens. Also, our data showed that this decline in intelectin-1 level increasing in obese patients than non-obese, as illustrated in Table 2.

PCOS women have been characterized by hyperinsulinemia, $\mathrm{IR}$, an increase in risk of glucose intolerance, diabetes especially type 2 and an increased prevalence of lipid-related abnormalities. The IR may play a pivotal role in the pathogenesis of PCOS, although the mechanisms underlying PCOS in women are not completely understood. Furthermore, independent of obesity, the presence of hyperinsulinemia in women with PCOS patients was previously confirmed [11]. On the other hand, intelectin-1 levels change during the menstrual cycle in women. Also, in a recent study, researchers reported that a significant difference $(\mathrm{P}<0.001)$ in the level of intelectin-1 between regular menstrual cycle and irregular menstrual cycle in PCOS patients, as illustrated in Table 2. Hence, it may be considered a reliable scientific proof that from the first to fifth days of the cycle intelectin-1 levels were more stable compared to those produced later in the cycle [12]. The important physiological role of intelectin-1 in metabolism of glucose, intelectin's target tissues, a receptor, or pathways of relevant signal transduction are still to be determined and further work must be carried out. Although, there are some scientific studies, in vitro, reported that intelectin-1 may has an ability to increases insulin signal transduction via activating the protein kinase Akt/protein kinase B and enhancing insulin-stimulated glucose transport in isolated human adipocytes [13]. Moreover, it is too clear that adiposity has a large role in the generating and maintaining of PCOS in women. Also, it is demonstrated that obesity worsens the phenotype of reproductive and metabolic in many women with PCOS. Furthermore, because intelectin-1 circulates in blood, it may also act at distant sites, e.g., muscle, liver, and subcutaneous fat, as well as may increase after weight loss-induced improvements in sensitivity of insulin and glucose metabolism, and thus may play a wider role in nutrient storage and partitioning. Hence, regulate and control of obesity in women patients with PCOS may also help to control IR and improve the metabolic parameters [11].

IR can be defined as a form of biological misinformation the body in which the insulin hormone receptors on the cell membrane are appropriately not responding to the insulin, thus the glucose in blood cannot get into cells, which creates a hypoglycemic reaction. This situation makes the pancreas pump out high doses of insulin to try to get the glucose out of blood 


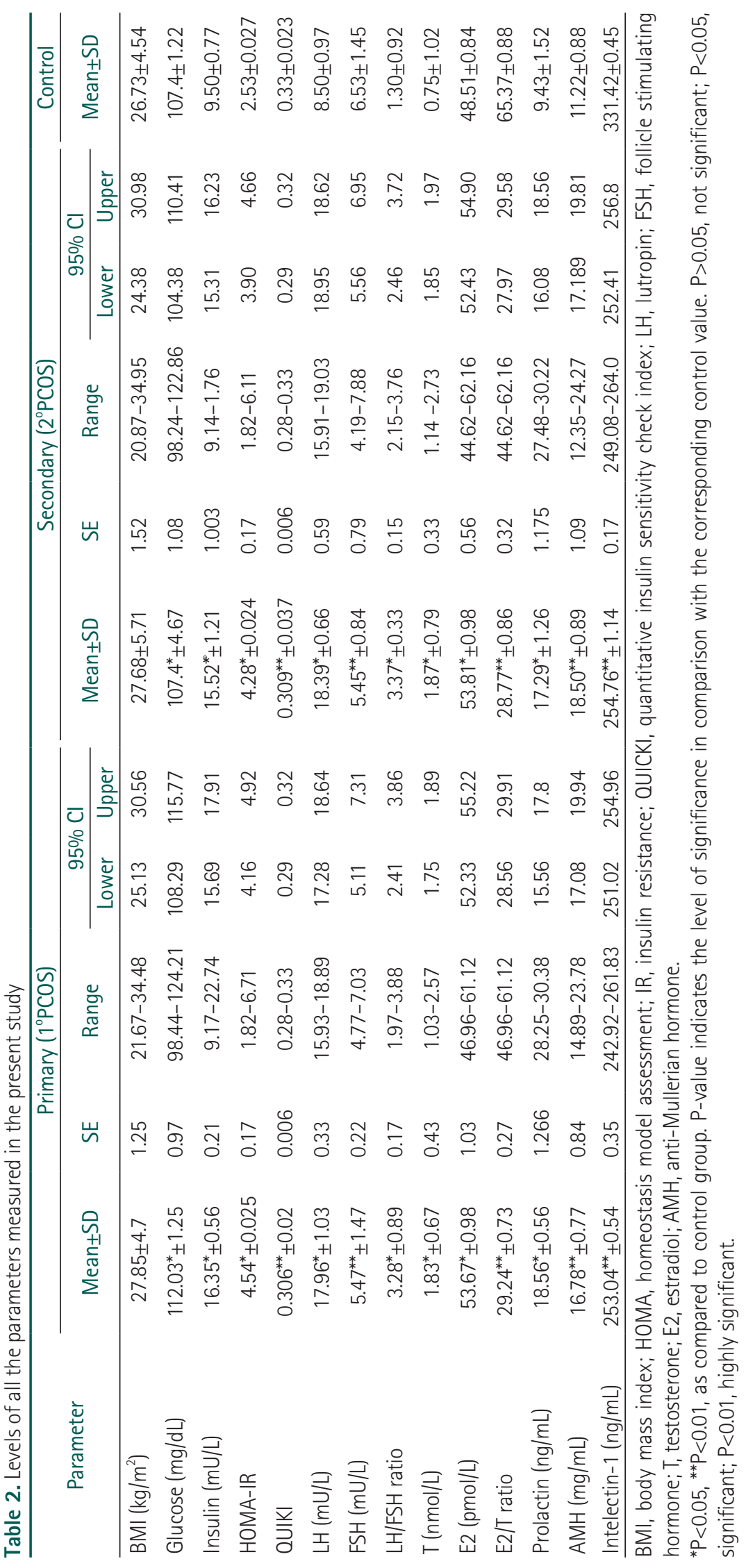


and into cells, so decreased of the ability of insulin hormone to regulate and to signal changes the levels of glucose in the blood and perhaps arises IR [14]. In women patients with PCOS, the mechanisms for significantly increased of IR appears to be too complex, thus the action of insulin must be clarified before starting out. The Insulin hormone may bind itself to its cell surface receptor that has homologous structure to the insulin-like growth factor-1 (IGF-1) receptor. The Insulin hormone stimulates the tissue cells to glucose uptake via increasing the translocation of the insulin responsive glucose transporter 4 from intracellular vesicles to the cell surface in tissues. The occurrence of this pathway may require sharing and mediated of phosphatidylinositol 3-kinase. On the other hand, the MAPK-ERK pathway is mediating in the growth and differentiation of cells because it is able to stimulate a cascade of enzymes, including serine/threonine, Raf, MAPK and MAPK-ERK1/2 of the non-classical models [3]. QUICKI has been developed to improve of the sensitivity and accuracy of fasting measurements. There are a well association between QUICKI and clamp measurements in patients with higher BMI and the lean. Also, it may also show a significantly correlation with HOMA-IR due to infeasibility of euglycemic insulin clamp method, across globe HOMA-IR method is represented as a gold standard test for assay of IR in clinical practice and population-based research studies [15]. In this work (Table 3), increased level of IR in patient's women with PCOS may be due to mechanism of action between the insulin hormone and its receptors in ovaries which have been demonstrated their capable of stimulating ovarian growth and steroid genesis, increased intra ovarian androgens, disrupts normal follicular genesis, and development of multiple ovarian cysts and ovarian enlargement, thus mechanisms for hyper-insulinemia include functional problems in the insulin. The significantly increased of IR is may lead to decreased uptake, and increased release, of free fatty acids from adipose tissue, so increased triglyceride synthesis and in turn promotes the assembly and secretion of triglyceride containing very low density lipoprotein (VLDL) [16]. On the other hand, IR may impair VLDL particle clearance which leading to greater interchange of core triglyceride from VLDL with LDL and HDL, LDL-TG and HDL become substrates for hepatic tissue lipase leading to smaller as well as more denser particles. Therefore, significantly increased of free fatty acids levels may cause down regulation of the ABCA 1 transporter, so lead to it involved in reverse cholesterol transport [3]. Also, significantly hyperinsulinemia as in rare syndromes of type A or B IR could lead to insulin "spillover" activation of ovarian IGF-1 receptors, thus stimulate the production of androgen. In addition, hyperinsulinemia has an ability to inhibit synthesis of sex hormonebinding globulin in the liver and results in significantly increased of blood free $\mathrm{T}$ levels. Furthermore, the hyperinsulinemia state can lead to a significantly decrease in stage of secretion of IGF binding protein -1 in both the liver and the ovary tissues, so increased intraovarian bioavailability of two important regulators IGF-1 and IGF-2 of ovarian follicular maturation and steroidogenesis. There are some studies postulated that clinical features of PCOS in women patients may come from geneti-

Table 3. The levels of insulin resistance parameters

\begin{tabular}{|c|c|c|c|c|}
\hline Group & Insulin (mU/L) & Glucose (mg/dL) & $\mathrm{HOMA}-\mathrm{IR} \pm \mathrm{SD}$ & $\mathrm{QUIKI} \pm \mathrm{SD}$ \\
\hline \multicolumn{5}{|l|}{ Control } \\
\hline Obese $(n=27)$ & $10.81 \pm 1.37$ & $110.35 \pm 3.41$ & $2.94 \pm 0.03$ & $0.325 \pm 0.04$ \\
\hline \multicolumn{5}{|l|}{$1^{\circ} \mathrm{PCOS}$} \\
\hline Obese $(n=28)$ & $17.75^{*} \pm 1.11$ & $117.32^{*} \pm 3.74$ & $5.14^{*} \pm 0.07$ & $0.301 \pm 0.02$ \\
\hline \multicolumn{5}{|l|}{$2^{\circ} \mathrm{PCOS}$} \\
\hline Obese $(n=31)$ & $16.87^{*} \pm 1.13$ & $116.78^{*} \pm 4.83$ & $4.86^{*} \pm 0.03$ & $0.303 \pm 0.04$ \\
\hline Non-obese $(n=33)$ & $14.17^{*} \pm 1.04$ & $105.89^{*} \pm 3.16$ & $3.70^{*} \pm 0.06$ & $0.314 \pm 0.02$ \\
\hline
\end{tabular}

Values are presented as mean \pm SD.

HOMA, homeostasis model assessment; IR, insulin resistance; OUICKI, quantitative insulin sensitivity check index.

${ }^{*} \mathrm{P}<0.05,{ }^{*} \mathrm{P}<0.01$, as compared to control group. 
cally determined hypersecretion of androgens by the ovary that perhaps starts during the foetal period as a result of significantly elevated of androgen exposure, either during the vulnerable period or at puberty [17]. Other important scientific research reported that, excess androgens exposure during the foetal development and growth can affect on the programming of the hypothalamo-pituitary unit or hormonal-cascade system that results in hypersecretion LH, higher BMI and development of abdominal obesity with consequent IR as well as anovulation [16]. Furthermore, It was demonstrated that women with PCOS patients have significantly levels of oxidative stress which may be result from hyperglycemia and higher levels of free fatty acid leads to excess production of ROS, IR, and chronic inflammation by producing TNF $\alpha$ from multinucleated cells. Moreover, the correlation between IR and PCOS in patient's women may results in several metabolic complications such as atherosclerosis, risk for developing gestational diabetes and type 2 diabetes mellitus, hypertension, atherogenic dyslipidemia, hypercoagulability, and vascular endothelial dysfunction, all risk factors for cardiovascular disease. The studies revealed that PCOS patient's women have a sevenfold increased risk for myocardial infarction as well as increased cardiovascular risk independent of obesity [3].

LH and FSH are the hormones secreted from the pituitary gland in response to stimulation by gonadotropin releasing hormone $(\mathrm{GnRH})$ from the brain and that have an ability to encourage ovulation therefore their levels varies throughout the menstrual cycle, and it appears that different patterns of $\mathrm{GnRH}$ pulses from the brain affect the different patterns of $\mathrm{LH}$ and FSH secretion. The role of $\mathrm{LH}$ is to control the production of female hormones (estrogen and progesterone) in the ovary, while FSH role is represented on the controls of development and release of eggs in the ovary [2]. Before we interpret the results we would like to point out key point is that after ovulation, levels of estrogen and progesterone may be elevated which result in decreased the level of the pulses of GnRH and this slowing of $\mathrm{GnRH}$ allows for later FSH secretion (at the time of menstruation), which normally leads to egg development in the next cycle. On the other hand, if the pulses of GnRH are not slowed down, subsequent FSH production is diminished. One can therefore see that this ability to slow GnRH pulses seems to be quite important for subsequent FSH secretion, and therefore subsequent development of an egg [18]. So, in PCOS patients the increased in level of LH hormone and decreased level of FSH hormones might be due to that GnRH secretion is relatively fast, and that does not slow down very well in response to estrogen and progesterone, as illustrated in Table 4. Furthermore, this may help to explain why PCOS patients frequently do not ovulate. Women with PCOS often have high levels of LH secretion which lead to the high levels of androgens (male hormones such as T), along with low levels of FSH contributes to poor egg development and an inability to ovulate. A lack

Table 4. The levels of intelectin-1, AMH and some endocrinological hormones in women of healthy control and PCOS patients

\begin{tabular}{|c|c|c|c|c|c|c|}
\hline \multirow{3}{*}{ Parameters } & \multicolumn{4}{|c|}{ Women patients with PCOS } & \multirow{2}{*}{\multicolumn{2}{|c|}{ Control $(n=56)$}} \\
\hline & \multicolumn{2}{|c|}{ Primary $\left(1^{\circ} \mathrm{PCOS}\right)(\mathrm{n}=60)$} & \multicolumn{2}{|c|}{ Secondary $\left(2^{\circ} \mathrm{PCOS}\right)(n=64)$} & & \\
\hline & Obese $(n=28)$ & Non-obese $(n=32)$ & Obese $(n=31)$ & Non-obese $(n=33)$ & Obese $(n=27)$ & Non-obese $(n=29)$ \\
\hline Intelectin-1 (ng/mL) & $249.36^{* *} \pm 1.55$ & $256.73^{* *} \pm 2.37$ & $251.74^{* *} \pm 1.45$ & $257.78^{* *} \pm 1.67$ & $327.75 \pm 2.16$ & $335.09 \pm 1.04$ \\
\hline AMH (mg/mL) & $19.74^{* *} \pm 1.55$ & $17.38^{* *} \pm 1.21$ & $20.03^{* *} \pm 1.32$ & $16.97^{* *} \pm 1.08$ & $12.43 \pm 1.33$ & $10.02 \pm .25$ \\
\hline $\mathrm{FSH}(\mathrm{mU} / \mathrm{mL})$ & $5.42^{*} \pm 0.94$ & $5.52^{*} \pm 1.07$ & $5.34^{*} \pm 0.96$ & $5.57^{*} \pm 0.80$ & $6.35 \pm 1.20$ & $6.71 \pm 1.20$ \\
\hline E2 (pmol/L) & $55.28^{*} \pm 1.8$ & $52.07^{*} \pm 1.45$ & $55.64^{*} \pm 1.52$ & $51.98^{*} \pm 0.81$ & $49.87 \pm 1.55$ & $47.16 \pm 2.10$ \\
\hline $\mathrm{T}(\mathrm{nmol} / \mathrm{L})$ & $1.89^{*} \pm 0.03$ & $1.78^{*} \pm 0.05$ & $1.93^{*} \pm 0.03$ & $1.81^{*} \pm 0.06$ & $0.87 \pm 0.03$ & $0.64 \pm 0.07$ \\
\hline E2/T ratio & $29.23^{* *}$ & $29.22^{* *}$ & $28.81^{* *}$ & $28.70^{* *}$ & 57.27 & 73.48 \\
\hline PRL (ng/mL) & $18.97^{*} \pm 0.16$ & $14.59^{*} \pm 1.12$ & $19.24^{*} \pm 2.40$ & $15.33^{*} \pm 1.17$ & $10.54 \pm 0.36$ & $8.33 \pm 0.57$ \\
\hline
\end{tabular}

Values are presented as mean \pm SD.

AMH, anti-Mullerian hormone; PCOS, polycystic ovary syndrome; FSH, follicle stimulating hormone; LH, luteinizing hormone; E2, estradiol; T, testosterone; PRL, prolactin.

${ }^{*} \mathrm{P}<0.05$, ${ }^{*} \mathrm{P}<0.01$, as compared to control group. 
of ovulation also leads to relative deficiencies of progesterone production by the ovary, which often leads to absence of menstrual periods. On the other hand, the role of LH in PCOS women is support theca cells in the ovaries that provide androgens and hormonal precursors for E2 production. Hence, the detrimental impact of endocrinological disorder, which is linked to LH hyper-secretion and dysfunction of ovulatory, is may be attributed to increased LH levels. Therefore, the higher levels of LH than normal in a woman may clearly mean that the ovaries are absent or not functioning so that that puberty is early and its height may be causes infertility [19]. Also, the increase in serum E2 levels may be cause a decrease in FSH production by inhibiting the production of $\mathrm{GnRH}$ in the hypothalamus. There are many evidences that a moratorium on the development of follicles in women with PCOS which is highly observed may reflect the disrupted function of the endocrine environment. These reports have also shown that precocious puberty of many ovarian folliculesin may increase in polycystic ovaries due to granulosa cells of these follicles are less sensitive to LH and their size is smaller than other follicles with normal cycle and produces abnormal amounts of E2 which lead to inhibits FSH levels and hinders the healthy follicular maturation [2]. Our study shows that in PCOS women high LH/FSH ratio is a common occurrence, although this LH/FSH ratio is not characteristic attribute of all PCOS women. Most of the PCOS women with normal gonadotrophin ratio belong to a group of patients suffering from hyperinsulinemia and obesity [19]. The traditional belief that obesity plays a serious role in the pathophysiology of PCOS dated back to years of Stien Leventhal but the puzzling fact that not all PCOS women are obese, moreover, not every woman with PCOS has an abnormal LH/ FSH ratio nor they all possess the hormonal and biochemical changes suggested to the disease. This diversity in disease criteria inspired us to think about measuring correlations between disease manifestations and to answer the question "does higher BMI necessarily indicate a higher LH/FSH ratio or greater incidence of hirsute or menstrual disturbance" [18]. There are some reports indicating that there is no cure for PCOS and it does not go away on its own, while the drugs in circulation are aimed at reducing its symptoms and prevent further complications. In other words, there are some options that should be adopted by patient's women depending on the type and severity of individual women's symptoms and their desire to become pregnant. Of these options, diet, exercise and maintaining a healthy body weight which may help in reduce level of IR as well as weight reduction can also reduce the level of $\mathrm{T}$, insulin and LH levels. This has a significant role in managing the symptoms of PCOS in women with PCOS [2].

E2 is an essential hormone that produced in endocrine and non-endocrine tissues such as fat, liver, adrenal, breast, and neural tissues, as well as its produced in especially within the ovary follicles of the women. It has a necessary role for the maintenance and development of women reproductive tissues such as breasts, uterus, and vagina in the times of puberty, adulthood, and pregnancy.

On the other hand, $\mathrm{T}$ hormone is the main circulating active androgen, and the level of total serum $\mathrm{T}$ is the first-line recommendation for assessing androgen excess in women and is the most direct evidence for androgen excess [20]. Elevation level of $\mathrm{E} 2$ hormone is may be due to the E2 metabolism by the uterus is abnormal in women with PCOS. Nevertheless, it is not surprising that elevated E2 levels are typically found in obesity women with PCOS. The adipose tissues represent an important peripheral site of aromatase enzyme activity, also, it may serve as the second source of estrogen production via aromatase enzyme that converts androstenedione to E2 and E1 as well as it is the major source of circulating estrogens in women after menopause. On the other hand, IR or hyperinsulinemia is may also represent a major cause of hyperandrogenism (increased $\mathrm{T}$ level) in PCOS women, through stimulating the ovarian to increased secretion of androgen and inhibiting the production of hepatic sex hormone-binding globulin [21].

The E2/T ratio may provide an important information about the activity of aromatase enzyme because conversion of androgens to estrogens is mediated by CYP19, suggesting that the $\mathrm{E} 2 / \mathrm{T}$ ratio may represent a direct biomarker of aromatase activity. In the polycystic ovary, the androgens may synthesize by theca cells more than the corresponding cells in a normal ovary. In contrast, the activity of aromatase is lower in granulosa cells of the polycystic ovary, which lead to an imbalance in the production of estrogen and androgen [22]. So, this cannot preclude the importance of an aromatase activity disorder in the etiology of PCOS in women (with primary and secondary type), as there may exist causative mutations in the untranslated regions or within introns. On the other hand, there is some evidence that obesity, especially abdominal obesity, may lead to 
exacerbate both the clinical and endocrine features of PCOS in women which demonstrate that IR consider significantly more serious in these patients than normal-weight counterparts [20]. Furthermore, there are some scientific reports that estrogen has the more capacity to favorably regulate of both body composition and homeostasis of glucose to prevent diet-induced obesity. Moreover, the expression of aromatase in the ovarian follicle is may also responsible for the cyclic changes in levels of serum E2 and the modulation of the structure and function of the female reproductive tract and is essential for the survival, fertilization and implantation of oocytes and this demonstrate that PCOS promotes a hyperestrogenic state [20]. In the present study, PCOS with high E2 levels may lead to more serious of hyperandrogenism but with relatively decreased in levels of E2/T. So, this agrees with another previous study showing that higher levels of E2 may be caused by increased RNA expression of granulosa cell aromatase and its activity [21]. Nevertheless, higher levels of T in PCOS patient's women may cause inhibited aromatase activity. Thus, the androgen can dosedependently directly affect on aromatase activity, or indirectly by regulating other factors such as E2 and LH. Some report suggests that early exposure of women to androgen may induce sex-specific organizational changes of aromatase expression in the preoptic area [22]. The role of PRL in PCOS women is still controversial.

In the present study (Table 4), PCOS women had significantly higher level of PRL as compared to control group which may lead to induce suppression of the hypothalamic-pituitarygonadal axis, resistance of the ovary to gonadotropin action and results in amenorrhea and anovulation. In the ovary, PRL may block the folliculogenesis and inhibits the activity of aromatase enzyme in granulosa cell, which leading to hypoestrogenism and an ovulation. Thus, increased level of PRL may led to adversely acts the fertility potential by impairing pulsatile secretion of $\mathrm{GnRH}$ and hence interfering with ovulation [23]. The PRL may have an ability to stimulate insulin and suppress adiponectin in addition to stimulate release of interleukin-6 which further suggests to an important role in the manifestation of high levels of adipokines exacerbate IR and impair insulin secretary capacity [24]. It may be role to stimulating increased IR by down-regulation of insulin receptors and/or due to defects of post receptors. There are several factors involve in the neuroendocrine control of PRL secretion from the anterior pi- tuitary gland which including PRL releasing factors and PRLrelease inhibiting factors, may be exert a tonic inhibitory control [25]. The main physiological PRL release inhibiting factors is dopamine which secreted from the dopaminergic neurons of hypothalamus. Elevated level of insulin resistant state lead to decline in dopamine levels and increase to the normal range following the return to the insulin sensitive state [26]. Scientific studies reported that there is a high interaction between energy homeostasis and the dopamine receptor D2 variants. These reports revealed that the polymorphism of Ser311Cys which impairs the dopamine receptor D2 signal transduction pathways is may correlated with an increased in obesity and decreased in resting energy expenditure in human [27]. On the other hand, higher level of BMI or the obese in PCOS women may be have the possible effect on the pathogenesis of elevated level of serum PRL, which should be taken in considered in cases of the idiopathic increased. Also, release of PRL spontaneously has been shown to be significantly increased in obese women in direct proportion to the size of the visceral fat mass. Moreover, activation of the dopamine D2 receptor lead to inhibited of PRL secretion, therefore its secretion may increase when the reduced in dopamine D2 receptor availability occur in the brain, which makes these individuals more likely to have elevated PRL secretion [28]. Finally, our results support the observations by Szosland et al. [29] who draw the following points. First, in PCOS women the incidence of significantly increased of blood PRL may be not more frequent in comparison to women of healthy control group; therefore, it should not be considered a feature of PCOS, as both are distinct clinical entities. Second, the possibility of incidence the significantly increased of PRL should be taken into consideration in every woman with suspicion of PCOS, may be due to some common clinical signs of both disorders. Third, each patient with diagnosed PCOS and significantly increased of PRL should be diagnosed towards other reasons of higher elevated of PRL in blood may be due to that the coexistence of these two pathological conditions is possible.

The present our study, however, did have limitation which may relate to the number of women patients studied. Hence, our results cannot represent the actual state of the entire patient group in Iraq due to the low volunteer number of patients and also depends on the cooperatively of patients and their willing to participate in the present study. On the other hand, although 
the present study could not yield a statistically significant pattern of the parameters observed which may be due to the relatively contribution of small number of PCOS women patients included, but we believed that it pointed to a connection between some of the parameters.

From the above study, it can be concluded that IR has an important etiological role in the pathophysiology of infertility through its effect on the levels of intelectin-1 and endocrinological hormones in patients women (with $1^{\circ} \mathrm{PCOS}$ and $2^{\circ} \mathrm{PCOS}$ ) which could be considered as good biomarkers for early detection of the development of vascular complications in PCOS patient's women. Therefore, patients with PCOS should adjust their diet and follow some strategies that can lower IR level, correct the level of serum intelectin -1 and rearrange metabolism in these women.

\section{References}

1. Spritzer PM, Lecke SB, Satler F, Morsch DM. Adipose tissue dysfunction, adipokines, and low-grade chronic inflammation in polycystic ovary syndrome. Reproduction 2015;149:R219-R227.

2. Le MT, Le VN, Le DD, Nguyen VQ, Chen C, Cao NT. Exploration of the role of anti-Mullerian hormone and LH/FSH ratio in diagnosis of polycystic ovary syndrome. Clin Endocrinol (Oxf) 2019;90:579-585.

3. Bannigida DM, Shivananda Nayak B, Vijayaraghavan R. Insulin resistance and oxidative marker in women with PCOS. Arch Physiol Biochem 2018 Nov 17 [Epub]. https://doi.org/10.1080/1 3813455.2018 .1499120

4. Kenawi MZ, Akl EM, Sabry JH, Mostafa ST. Evaluation of serum level of omentin-1 in females with hirsutism. J Cosmet Dermatol 2019 Jun 13 [Epub]. https://doi.org/10.1111/jocd.13043

5. Tang YL, Yu J, Zeng ZG, Liu Y, Liu JY, Xu JX. Circulating omentin-1 levels in women with polycystic ovary syndrome: a metaanalysis. Gynecol Endocrinol 2017;33:244-249.

6. Infertility workup for the women's health specialist: ACOG Committee Opinion, number 781. Obstet Gynecol 2019;133:e377e384.

7. Gutch M, Kumar S, Razi SM, Gupta KK, Gupta A. Assessment of insulin sensitivity/resistance. Indian J Endocrinol Metab 2015;19:160-164.

8. Ekka DC, Jain A, Puri M. Role of hormones in unexplained infertility. Indian J Med Biochem 2016;20:34-37.

9. Callahan TL, Caughey AB. Puberty, the menstrual cycle, and the menopause: infertility and assisted reproductive technologies. In: Callahan TL, Caughey AB. Blueprints obstetrics and gynecology. 7th ed. Philadelphia: Wolters Kluwer Health; 2018. p.212222.

10. Zhou Y, Zhang B, Hao C, Huang X, Li X, Huang Y, et al. Omentin-
A novel adipokine in respiratory diseases. Int J Mol Sci 2017;19: E73.

11. Guzel EC, Celik C, Abali R, Kucukyalcin V, Celik E, Guzel M, et al. Omentin and chemerin and their association with obesity in women with polycystic ovary syndrome. Gynecol Endocrinol 2014;30:419-422.

12. Guvenc Y, Var A, Goker A, Kuscu NK. Assessment of serum chemerin, vaspin and omentin-1 levels in patients with polycystic ovary syndrome. Int Med Res 2016;44:796-805.

13. Kareem JM, Hashim ZH, Almoayed HA. The relation of serum omentin-1 level with insulin resistance in patients with polycystic ovary syndrome and its relation with metformin treatment. Iraqi JMS 2017; 15:327-338.

14. Barbosa G, Cunha de Sa LB, Rocha DR, Arbex AK. Polycystic ovary syndrome (PCOS) and fertility. Open JEndocr Metab Dis 2016;6:58-65.

15. Ambiger S. Study of insulin resistance and lipid profile in polycystic ovarian syndrome. Int J Sci Res Publ 2016;6:1-5.

16. Kumar AN, Naidu JN, Satyanarayana U, Anitha M, Ramalingam $\mathrm{K}$. Association of insulin resistance and serum 25-OH vitamin$\mathrm{D}$ in Indian women with polycystic ovary syndrome. Int J Clin Biochem Res 2015;2:22-26.

17. Cakir E, Topaloglu O, Colak Bozkurt N, Karbek Bayraktar B, Gungunes A, Sayki Arslan M, et al. Insulin-like growth factor 1, liver enzymes, and insulin resistance in patients with PCOS and hirsutism. Turk J Med Sci 2014;44:781-786.

18. Chaudhari N, Dawalbhakta M, Nampoothiri L. GnRH dysregulation in polycystic ovarian syndrome (PCOS) is a manifestation of an altered neurotransmitter profile. Reprod Biol Endocrinol 2018;16:37.

19. Lal L, Bharti A, Perween A. To study the status of LH: FSH ratio in obese and non-obese patients of polycystic ovarian syndrome. IOSR J Dent Med Sci 2017;16:20-23.

20. Chen J, Shen S, Tan Y, Xia D, Xia Y, Cao Y, et al. The correlation of aromatase activity and obesity in women with or without polycystic ovary syndrome. J Ovarian Res 2015;8:11.

21. Shaw ND, Srouji SS, Welt CK, Cox KH, Fox JH, Adams JM, et al Evidence that increased ovarian aromatase activity and exdkanpression account for higher estradiol levels in African American compared with Caucasian women. J Clin Endocrinol Metab 2014;99:1384-1392.

22. Gonzalez B, Ratner LD, Scerbo MJ, Di Giorgio NP, Poutanen M, Huhtaniemi IT, et al. Elevated hypothalamic aromatization at the onset of precocious puberty in transgenic female mice hypersecreting human chorionic gonadotropin: effect of androgens. Mol Cell Endocrinol 2014;390:102-111.

23. Indira M, Babu CS. Estrogen, progesterone and serum prolactin levels in infertility cases of women. IOSR J Dent Med Sci 2015;14: 21-26.

24. Khalaf BH. The effect of long term administration of metformin on prolactin level and C-reactive protein in newly diagnosed level and women with type II diabetes mellitus. World J Pharm Pharm Sci 2015;4:156-169. 
25. Al-Fartosy AJ, Awad NA, Mahmood RA. A comparison study of leptin, oxidant/antioxidant status and some trace elements in women of healthy control and unexplained infertility in BasrahIraq. Indones Biomed J 2019;11:327-337.

26. Kanwar G, Jain N, Shekawat M, Sharma N. Estimation of LH, $\mathrm{FSH}$, prolactin and TSH levels in polycystic ovarian syndrome and correlation of LH and FSH with serum TSH levels. IOSR J Dent Med Sci 2015;14:64-68.

27. Grachev P, Li XF, Goffin V, O'Byrne KT. Hypothalamic prolactin regulation of luteinizing hormone secretion in the female rat. Endocrinology 2015;156:2880-2892.

28. Hasan JS, Ali AH, Baqir M, Fakhrildin MB. Role of prolactin in Iraqi obese women with polycystic ovary syndrome. Am J Med Med Sci 2015;5:135-139.

29. Szosland K, Pawlowicz P, Lewinski A. Prolactin secretion in polycystic ovary syndrome (PCOS). Neuro Endocrinol Lett 2015;36: 53-58. 